\title{
Ações do enfermeiro na prevenção da gravidez na adolescência na Atenção Básica
}

\author{
Nurse's action in the prevention off teenage pregnancy in Primary Care \\ Actuaciones del enfermero em la prevención del embarazo adolescente em Atención Primaria
}

Recebido: 07/01/2022 | Revisado: 13/01/2022 | Aceito: 16/01/2022 | Publicado: 18/01/2022

Eliane Rodrigues da Silva

ORCID: https://orcid.org/0000-0002-6989-3068 Universidade Iguaçu, Brasil

E-mail: eliane.silva590.irs@gmail.com

Mônica Guimarães da Silva

ORCID: https://orcid.org/0000-0003-2916-8571 Universidade Iguaçu, Brasil

E-mail: monika.silvaa@ outlook.com

Bruna Porath Azevedo Fassarella

ORCID: https://orcid.org/0000-0002-1400-4147 Universidade Iguaçu, Brasil

E-mail: brunaporath@gmail.com

Larissa Christiny Amorim dos Santos

ORCID: https://orcid.org/0000-0002-9705-5811

Universidade Iguaçu, Brasil

E-mail: amorimlari224@gmail.com

Raiane Monteiro Rodrigues da Silva

ORCID: https://orcid.org/0000-0002-1717-7254

Universidade Iguaçu, Brasil

E-mail: raiimonteiroo@gmail.com

Vitória da Silva Araújo

ORCID: https://orcid.org/0000-0003-2960-1347

Universidade Iguaçu, Brasil

E-mail: vitoriasilva178@gmail.com

Wanderson Alves Ribeiro

ORCID: https://orcid.org/0000-0001-8655-3789 Universidade Iguaçu, Brasil

E-mail: nursing_war@hotmail.com

Keila do Carmo Neves

ORCID: https://orcid.org/0000-0001-6164-1336 Universidade Iguaçu, Brasil

E-mail: keila_arcanjo@hotmail.com

Ana Lúcia Naves Alves

ORCID: https://orcid.org/0000-0003-0791-5775 Universidade Iguaçu, Brasil

E-mail: ananaves.alna@gmail.com

Fernando Salgado do Amaral

ORCID: https://orcid.org/0000-0003-4370-3198 Universidade Iguaçu, Brasil

E-mail: fernando.sal.81@gmail.com

\begin{abstract}
Resumo
No cenário atual a gravidez e um processo que faz parte da vida de várias mulheres, entretanto a gravidez precoce pode trazer danos a sua saúde da adolescente e de sua família, tendo em vista que é considerado um problema de saúde pública e social, pois a adolescência e um período de transformações físicas, psicológicas, hormonais e descobertas , a educação sexual deve ser iniciada o mais cedo possível, sendo assumida pelos pais, e complementada pela escola e profissionais de saúde, o enfermeiro possui um papel essencial no desenvolvimento de habilidades de prevenção e educação com os adolescentes. Este estudo tem como objetivo geral analisar o trabalho do enfermeiro na prevenção da gravidez na adolescência. Trata-se de uma revisão bibliográfica, que tem como objetivo analisar o trabalho do enfermeiro na prevenção da gravidez na adolescência. Posteriormente à leitura reflexiva dos artigos emergiram duas categorias: Ações do enfermeiro para auxiliar na prevenção da gravidez na adolescência e orientações do enfermeiro na sensibilização da prevenção da gravidez na adolescência. Para seleção da amostra, houve recorte temporal de 2015 a 2020, pois o estudo capturou as publicações dos últimos 5 anos. Conclui-se que o presente estudo evidenciou que o enfermeiro na atenção básica e fundamental para implementar ações estabelecendo estratégias que visem à prevenção da gravidez na adolescência.
\end{abstract}

Palavras-chave: Enfermagem; Gravidez na adolescência; Prevenção. 


\begin{abstract}
In the current scenario, pregnancy is a process that is part of the lives of many women, however, early pregnancy can harm the health of the adolescent and her family, considering that it is considered a public and social health problem, as the adolescence and a period of physical, psychological, hormonal and discovery transformations, sex education should be started as soon as possible, being assumed by parents, and complemented by school and health professionals, the nurse has an essential role in the development of skills. prevention and education with adolescents. This study has as general objective to analyze the work of nurses in preventing teenage pregnancy. This is a literature review, which aims to analyze the work of nurses in preventing teenage pregnancy. After the reflective reading of the articles, two categories emerged: Nurse actions to help prevent teenage pregnancy and nurses' guidance in raising awareness of the prevention of teenage pregnancy. For sample selection, there was a time frame from 2015 to 2020 , as the study captured publications from the last 5 years. It is concluded that this study showed that nurses in primary care are essential to implement actions establishing strategies aimed at preventing teenage pregnancy.
\end{abstract}

Keywords: Nursing; Teenage pregnancy; Prevention.

\title{
Resumen
}

En el escenario actual, el embarazo es un proceso que forma parte de la vida de muchas mujeres, sin embargo, el embarazo precoz puede perjudicar la salud de la adolescente y su familia, considerando que se considera un problema de salud pública y social, como la adolescencia y un período de transformaciones físicas, psicológicas, hormonales y de descubrimiento, la educación sexual debe iniciarse lo antes posible, siendo asumida por los padres, y complementada por la escuela y los profesionales de la salud, la enfermera tiene un papel fundamental en el desarrollo de habilidades. prevención y educación con adolescentes. Este estudio tiene como objetivo general analizar la labor del enfermero en la prevención del embarazo adolescente. Se trata de una revisión de la literatura, que tiene como objetivo analizar el trabajo de las enfermeras en la prevención del embarazo adolescente. Luego de la lectura reflexiva de los artículos, surgieron dos categorías: acciones de enfermeras para ayudar a prevenir el embarazo adolescente y orientación de las enfermeras en la sensibilización sobre la prevención del embarazo adolescente. Para la selección de la muestra, hubo un período de tiempo de 2015 a 2020, ya que el estudio capturó publicaciones de los últimos 5 años. Se concluye que este estudio mostró que las enfermeras de atención primaria son fundamentales para implementar acciones que establezcan estrategias encaminadas a prevenir el embarazo adolescente.

Palabras clave: Enfermería; Embarazo en la adolescencia; Prevención.

\section{Introdução}

No decorrer da formação acadêmica, tivemos acesso a diversos artigos científicos falando sobre a temática da adolescência, tanto para compor a discussão de algumas disciplinas quanto na construção de trabalhos que tinham relação com a temática. Sendo assim, ao pensarmos na construção da pesquisa em tela, nos remetemos a essa temática, visto que falar de adolescência associado a prevenção da gravidez é algo extremamente necessário para população como todo.

Conforme Fiedler et al. (2015, p. 9), a adolescência é um período da vida do ser humano caracterizado por transformações social, psicológica, anatômicas e hormonais que juntamente com as novas experiências vivenciadas, definem a construção da personalidade de um futuro adulto.

De acordo com o Estatuto da Criança e do Adolescente (ECA), a adolescência é conceituada com a faixa etária de 12 a 18 anos.

O estudo de Rocha et al., (2017, p. 4) nos informa que o enfermeiro desempenha um fundamental papel na Equipe de Saúde da Família (ESF), podendo promover ações interdisciplinares que integrem família, escola e comunidade, na qual se despertará no adolescente o interesse de ampliar o conhecimento sobre as transformações do corpo, a busca por prazer, quebrando 'tabus' e desenvolvendo habilidades como a escuta qualificada, criação de vínculo e flexibilização na captação desse jovem para o serviço de saúde, que contribui para o seu desenvolvimento de forma segura e saudável.

O enfermeiro é primordial à prática de ações de prevenção e promoção a saúde, desenvolvidas pela Estratégia da Saúde da Família. É indiscutível que o papel de educador e orientador é inerente ao enfermeiro (Rocha et al, 2017).

O hebiatra é uma especialidade médica voltada para o cuidado dos adolescentes. A fase da adolescência é caracterizada pelas transformações físicas, e psíquicas típicas do processo de crescimento e desenvolvimento humano. 
O hebiatra aborda questões muito importantes nessa fase da vida como sexualidade, drogas, primeiro beijo, menstruação, alimentação excessos de exercícios físicos, sedentarismo e muitas outras questões pertinentes a esse momento tão delicado da vida. Atua não só na parte médica, biológica, mas atua também na esfera social e psicológica (Costa, 2020).

Dentro das práticas vivenciadas no campo de estágio foram evidenciadas um número grande de adolescentes realizando pré-natal de alto risco, dentre essas adolescentes algumas com doença hipertensiva, podendo desenvolver outras patologias como; pré-eclâmpsia, doença hipertensiva específica da gestação (DHEG), síndrome de Hellp ou até mesmo descolamento prematuro da placenta (DPP).

A gravidez na adolescência é considerada um fato precoce para essa etapa da vida, conforme nos informa Borrel (2017, p. 4), resultando em serias complicações, como abandono das atividades escolares, riscos para o feto e para mãe, conflitos familiares, discriminação social, afastamento de grupos de convivência, adiamento ou destruição de sonhos, tristeza solidão, esses sentimentos podem surgir em consequências da gestação da adolescência.

O estudo de Oliveira et al., (2008, p. 5), destaca que a educação sexual deve começar o mais cedo possível, deve ocorrer de maneira contínua e estar vinculada a formação de todas as crianças e adolescentes, sendo iniciada e assumida pelos pais, complementada pela escola e profissionais de saúde. É fundamental que a equipe da Unidade de Saúde trabalhe a sexualidade pelo viés da autoestima, seja durante a consulta individual, seja nos grupos ou nas atividades de parceria com a comunidade e escolas.

Pensar a gravidez na adolescência e o papel do enfermeiro neste processo é algo necessário, visto que a gravidez na adolescência é considerada um problema de saúde pública que leva a grandes problemas econômicos, gastos de saúde, interrupções aos estudos, além de complicações durante a gravidez que podem ser desencadeadas tanto para a mãe, como para o recém-nascido (Ribeiro et al., 2017).

O enfermeiro possui um papel essencial no desenvolvimento de habilidades preventivas e educativas com os adolescentes estabelecendo estratégias que visem à prevenção da gravidez na adolescência, criando grupos com propósitos na promoção de saúde e prevenção de infecções sexualmente transmissíveis (ISTs) e gravidez precoce, visando orientar os jovens sobre a importância da participação ativa nas ações de educação em saúde, no intuito de que se tornem capazes de lidar com suas próprias decisões, e elencando atitudes positivas para lidar com papel do autocuidado (Gurgel et al. 2010).

Este estudo constitui-se de uma reflexão sobre o histórico das políticas públicas em saúde voltadas para adolescentes e jovens brasileiro. Discutiu-se especialmente, sobre o programa Saúde do adolescente (PROSAD), com atenção à para a população-alvo, o direcionamento das ações para atender os adolescentes a variabilidade das metas propostas e a equipe disponível para intervir na população

O tema da sexualidade adolescente gerou intenso debate na sociedade brasileira em janeiro de 2020, com a proposta de abstinência (ou adiamento da vida sexual) como política pública para o enfrentamento da gravidez na adolescência. O tópico entrou em pauta após anúncio do Ministério da Mulher, da Família e dos Direitos Humanos (MMFDH) sobre a elaboração de uma Política Nacional de Prevenção ao Risco da Atividade Sexual Precoce, om a criação da Semana Nacional de Prevenção da Gravidez na Adolescência, pelo Governo Federal, por intermédio da Lei no 13.7981, em janeiro de 2019, a ser comemorada anualmente na primeira semana de fevereiro, o MMFDH, em parceria com o Ministério da Saúde, iniciou uma campanha nacional de sensibilização de adolescentes sobre os efeitos indesejáveis da "gravidez precoce" (Silva, et al.,2020)

Atualmente temos diversas legislações no âmbito da Saúde, da Educação, da Assistência Social que priorizam crianças e adolescentes em programa e projetos, visto o que preconiza no ECA - Estatuto da Criança e do Adolescente em seu art. $3^{\circ}$ : 
A criança e o adolescente gozam de todos os direitos fundamentais inerentes à pessoa humana, sem prejuízo da proteção integral de que trata esta Lei, assegurando-lhes, por lei ou por outros meios, todas as oportunidades e facilidades, a fim de lhes facultar o desenvolvimento físico, mental, moral, espiritual e social, em condições de liberdade e de dignidade. (ECA,1990, p. 02).

$\mathrm{Na}$ direção da promoção da saúde do adolescente, em nosso país, é função da atenção primária implementar o acompanhamento do crescimento e do desenvolvimento, no qual inclui-se a abordagem à saúde reprodutiva de maneira integral e permanente, envolvendo educação em saúde, atividades em grupo e atendimento individual (Fiedler et al., 2015).

No entanto, observa-se que as ações são pontuais, prevalecendo apenas a distribuição de métodos. Pensando neste viés, faz-se necessário discutirmos a importância do papel do enfermeiro no processo de prevenção da gravidez na adolescência em uma unidade básica de saúde (Fiedler et al., 2015).

O estudo justifica-se, pois viabilizam a qualidade de vida das pessoas é algo fundamental, sendo o trabalho do enfermeiro neste âmbito é diversificado, pois, além do cuidado ao indivíduo abrange ações educativas, referentes a sexualidade (Rocha et al., 2017).

Gestação precoce nesse grupo populacional vem sendo considerada um problema de saúde pública. É comum e na maioria das vezes evitável e associado a sequelas negativas para as adolescentes que se tornam grávidas e para seus filhos. Podem acarretar complicações obstétricas, com repercussão para mãe e o recém-nascido, como problemas psicossociais e econômicas (Gurgel et al., 2010).

A gravidez na adolescência é considerada um problema de saúde Pública que leva a grandes problemas econômicos, gastos de saúde, interrupções aos estudos, além de complicações durante a gravidez que podem ser desencadeadas tanto para a mãe como para o recém-nascido, onde estratégias podem ser elucidadas visando minimizar estes problemas (Ribeiro et al., 2016).

Além de a gravidez na adolescência ser um problema social, tendo em vista que desencadeia uma série de outras intercorrências na vida dos jovens envolvidos - sobretudo, na vida da menina -, é também um problema de saúde pública. Muitas ficam grávidas novamente ainda na adolescência e estão propensas a ter hipertensão, partos prematuros, anemia, préeclâmpsia, desproporção feto-pélvico e restrição. Atualmente as ISTs no crescimento fetal, além de problemas consequentes de abortos provocados e/ou pela falta de assistência adequada (Taborda et al., 2014).

Mediante aos levantamentos, emergiram-se as seguintes questões norteadoras: Quais ações o enfermeiro implementa para auxiliar na diminuição do número de adolescente grávidas? Como o enfermeiro no papel de orientador e educador pode sensibilizar para evitar a gravidez na adolescência?

Este estudo, tem como objetivo geral analisar o trabalho do enfermeiro na prevenção da gravidez na adolescência e objetivos específicos entender estratégias elucidadas pelo enfermeiro para prevenção da gravidez na adolescência e descrever as orientações realizadas pelo enfermeiro aos adolescentes para prevenção da gravidez na adolescência.

\section{Metodologia}

Para desenvolvimento do estudo optou-se pela revisão de literatura na modalidade integrativa. A pesquisa de revisão integrativa envolve uma síntese de estudos científicos já produzidos em determinada área do conhecimento sobre o tema investigado, propiciando uma explicação detalhada dos elementos estudados (Marcolini; Lakatos, 2010).

Conforme orienta Mendes (2008) para a construção da revisão integrativa é necessário seguir seis etapas distintas: estabelecimento da questão norteadora; busca da literatura; definição das características do estudo; análise crítica dos estudos incluídos; interpretação dos resultados e apresentação da revisão integrativa. 
Na pesquisa em tela optou-se como base a realização de busca eletrônica em periódicos nacionais disponíveis na Biblioteca Virtual de Saúde (BVS), Literatura Latino e do Caribe em Ciências da Saúde (LILACS) e Scientific Electronic Library Online (SCIELO) e Google acadêmico, com pesquisas publicadas no período de 2015 a 2020, durante os meses de setembro e outubro de 2021. Os descritores utilizados para pesquisa foram: Enfermagem, gravidez na adolescência e prevenção.

Cabe destacar, que os critérios inclusão foram artigos com acessos íntegro, textos em formato de projeto, em língua portuguesa, que estejam nas datas estabelecidas nos critérios possuam articulação com o assunto abordado. Textos em língua estrangeira foram excluídos devido o interesse em embasar o estudo com dados do panorama brasileiro e os textos incompletos, para oferecer melhor compreensão através da leitura de textos na íntegra.

Após a associação de todas as palavras-chave, são encontrados 16.139 artigos, excluídos 16.043 e selecionados 27 artigos. Com a leitura dos artigos, após o critério de exclusão foram selecionados 20 artigos que abrangem o tema para a discussão.

\section{Resultados e Discussão}

Tabela 1 - Análise da busca:

\begin{tabular}{c|c|c}
\hline Descritores & Sites pesquisados & Artigos pós seleção de critérios \\
\hline \multirow{2}{*}{$\begin{array}{c}\text { Enfermeiro AND } \\
\text { Gravidez na adolescência AND } \\
\text { Prevenção }\end{array}$} & BDENF -23 artigos & 02 \\
\cline { 2 - 3 } & LILACS -100 artigos & 05 \\
\cline { 2 - 3 } & SCIELO -15 artigos & 03 \\
\cline { 2 - 3 } & GOOGLE Scholar -160 artigos & 10 \\
\hline
\end{tabular}

Fonte: Autores.

Quadro 1 - Síntese dos artigos incluídos:

\begin{tabular}{|c|c|c|c|c|c|}
\hline Ano & Tema & Fonte & Autor & Objetivo & Resultados/Conclusão \\
\hline 2015 & $\begin{array}{ll}\text { A prevenção } & \text { da } \\
\text { gravidez } & \text { na } \\
\text { adolescência } & \text { na } \\
\text { visão } & \text { de } \\
\text { adolescentes } & \end{array}$ & $\begin{array}{l}\text { Caderno } \\
\text { de saúde } \\
\text { pública }\end{array}$ & $\begin{array}{l}\text { WILDEMBU } \\
\text { RG et al. }\end{array}$ & 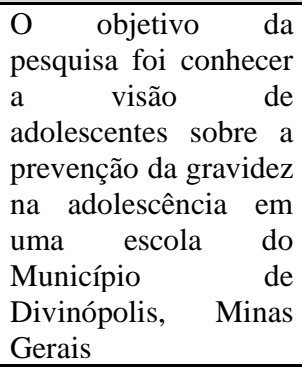 & $\begin{array}{l}\text { Resultados: percepção sobre a importância da } \\
\text { prevenção da gravidez na adolescência, } \\
\text { conhecimento sobre o uso dos métodos } \\
\text { contraceptivos, utilização dos métodos } \\
\text { contraceptivos e barreiras no acesso aos serviços } \\
\text { de saúde para prevenção da gravidez. } \\
\text { Conclusão: Conclui-se que os adolescentes } \\
\text { consideram a prevenção da gravidez na } \\
\text { adolescência como algo positivo. }\end{array}$ \\
\hline 2015 & 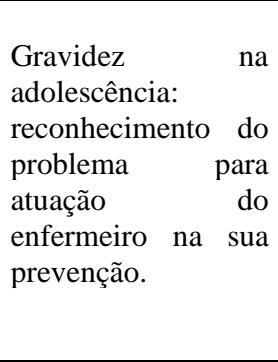 & $\begin{array}{l}\text { revista } \\
\text { Simpac }\end{array}$ & $\begin{array}{l}\text { MOREIRA,A } \\
\text {.T. et al. }\end{array}$ & $\begin{array}{lr}\text { Para } & \text { melhor } \\
\text { compreensão } & \mathrm{e} \\
\text { caracterização } & \text { do } \\
\text { problema } & \end{array}$ & $\begin{array}{l}\text { Resultados: adolescentes, entre } 10 \text { e } 19 \text { anos, } \\
\text { vem vivenciando uma gravidez não planejada, } \\
\text { tendo esse fator influência significativa no } \\
\text { abandono escolar, maior dependência dos pais ou } \\
\text { parceiros e uma dificuldade de inserção no } \\
\text { mercado de trabalho. } \\
\text { Conclusão: faz-se necessário focar o olhar no } \\
\text { comportamento das adolescentes, bem como } \\
\text { identificar possíveis situações precursoras da } \\
\text { gravidez. }\end{array}$ \\
\hline
\end{tabular}




\begin{tabular}{|c|c|c|c|c|c|}
\hline 2016 & $\begin{array}{lr}\text { Gravidez } & \text { na } \\
\text { adolescência } & \mathrm{a} \\
\text { importância } & \text { do } \\
\text { enfermeiro romo } & \text { como } \\
\text { educador proposto } \\
\text { de intervenção no } \\
\text { município e Buriti } \\
\text { Minas Gerais. }\end{array}$ & Una-sus & $\begin{array}{l}\text { ROCHA,M.C. } \\
\text { J. }\end{array}$ & $\begin{array}{l}\text { O objetivo deste } \\
\text { trabalho é elaborar um } \\
\text { projeto de intervenção } \\
\text { com o delineamento } \\
\text { de ações e estratégias } \\
\text { que possam contribuir } \\
\text { para o enfrentamento } \\
\text { da gravidez na } \\
\text { adolescência. . }\end{array}$ & $\begin{array}{l}\text { Resultado: Num contexto geral, o jovem } \\
\text { necessita aprender a viver e construir sua própria } \\
\text { identidade em um mundo caracterizado por } \\
\text { grandes contradições. A peculiaridade desta fase } \\
\text { favorece agravos de sua saúde física, emocional e } \\
\text { social, principalmente devido ao uso de drogas } \\
\text { lícitas e ilícitas, a violência, distúrbios de } \\
\text { sexualidade, doenças sexualmente transmissíveis } \\
\text { e gestação na adolescência }\end{array}$ \\
\hline 2016 & $\begin{array}{lrr}\text { Gravidez } & & \text { na } \\
\text { adolescência } & \text { e } & \text { o } \\
\text { papel } & & \text { da } \\
\text { enfermagem. } & & \end{array}$ & $\begin{array}{l}\text { Biblioteca } \\
\text { virtual em } \\
\text { saúde }\end{array}$ & GARCIA,A.R & $\begin{array}{l}\text { Relatar os impactos } \\
\text { que a gravidez na } \\
\text { adolescência. }\end{array}$ & $\begin{array}{l}\text { Resultados: . Este é um relato que discorre uma } \\
\text { vivência de estágio que ocorreu durante a } \\
\text { formação do curso Técnico em Enfermagem da } \\
\text { Escola GHC, em uma Unidade Básica de Saúde. } \\
\text { A gravidez na adolescência é uma questão que } \\
\text { preocupa os profissionais da saúde e da } \\
\text { educação, pelo impacto que traz na vida dos } \\
\text { jovens e no seu contexto familiar. Por outro lado, } \\
\text { nem sempre os adolescentes procuram a Unidade } \\
\text { Básica de Saúde para esclarecer dúvidas ou } \\
\text { buscar informações. }\end{array}$ \\
\hline 2016 & $\begin{array}{lr}\text { Gravidez } & \text { na } \\
\text { adolescência um } \\
\text { desafio para o } \\
\text { enfermeiro: revisão } \\
\text { de literatura }\end{array}$ & Una-Sus & $\begin{array}{l}\text { PEREIRA,K. } \\
\text { R. }\end{array}$ & $\begin{array}{l}\text { Realizar uma revisão } \\
\text { bibliográfica nacional } \\
\text { literatura nacion na } \\
\text { sobre a gravidez na } \\
\text { adolescência com } \\
\text { destaque para a } \\
\text { atuação do enfermeiro } \\
\text { e levantar nos estudos } \\
\text { selecionados. }\end{array}$ & $\begin{array}{l}\text { Resultados: Pela revisão verificou-se que o } \\
\text { profissional enfermeiro percebe que a gravidez } \\
\text { na adolescência, além de ser um problema de } \\
\text { saúde pública a concebe também um problema } \\
\text { social, influenciada pelos meios de comunicação } \\
\text { da sociedade e do ambiente familiar. } \\
\text { Conclusão: desses estudos apontam para a } \\
\text { necessidade de ações educativas sobre saúde } \\
\text { sexual e sexualidade para possibilitar as } \\
\text { adolescentes à oportunidade de escolhas de } \\
\text { métodos anticoncepcionais que lhes permitam ter } \\
\text { vida sexual sem correr o risco de uma gravidez } \\
\text { indesejada. }\end{array}$ \\
\hline 2016 & $\begin{array}{lr}\text { P } & \\
\text { revenção } & \text { da } \\
\text { gravidez } & \text { não } \\
\text { desejada } & \text { em } \\
\text { adolescentes: } & \\
\text { projeto } & \text { de } \\
\text { intervenção } & \text { na } \\
\text { atenção primária } & \end{array}$ & $\begin{array}{l}\text { Repositori } \\
\text { o UFMG }\end{array}$ & $\begin{array}{l}\text { VIEIRA,K.P. } \\
\text { et. Al }\end{array}$ & $\begin{array}{l}\text { Esse trabalho tem por } \\
\text { objetivo elaborar uma } \\
\text { proposta r de } \\
\text { intervenção que } \\
\text { contribua para } \\
\text { diminuir a incidência } \\
\text { de gravidez } \\
\text { indesejada junto as } \\
\text { adolescentes dessa } \\
\text { área de abrangência. }\end{array}$ & $\begin{array}{l}\text { Resultados: É alta a incidência de gravidez entre } \\
\text { as adolescentes cadastradas no território da } \\
\text { equipe verde da UBS do Vale do Jatobá situado } \\
\text { na Regional Barreiro em Belo Horizonte, Minas } \\
\text { Gerais. } \\
\text { Conclusão: justifica-se pela necessidade de } \\
\text { melhorar as informações sobre a prevenção da } \\
\text { gestação indesejada, tendo em vista que a } \\
\text { gravidez precoce contribui para o aumento de } \\
\text { intercorrências obstétricas e/ou neonatais, além } \\
\text { de repercussões sociais na vida dos adolescentes } \\
\text { e sua família. }\end{array}$ \\
\hline 2016 & \begin{tabular}{lr}
\multicolumn{2}{l}{ Papel do enfermeiro } \\
da estratégia & de \\
saúde da família & na \\
prevenção & da \\
gravidez & na \\
adolescência. &
\end{tabular} & $\begin{array}{l}\text { Revista } \\
\text { enfermage } \\
\mathrm{m} \text { centro } \\
\text { mineiro }\end{array}$ & SILVA et al. & 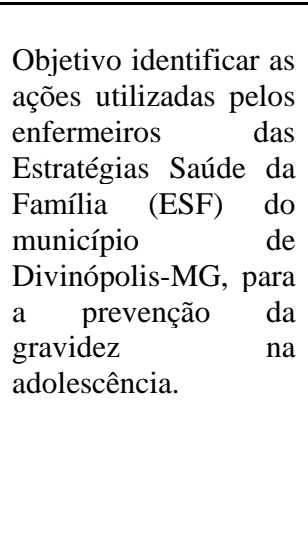 & $\begin{array}{l}\text { Resultados: Foram aplicados questionários aos } \\
\text { enfermeiros com } 14 \text { questões que nortearam a } \\
\text { prática desenvolvida ou não pelos enfermeiros } \\
\text { das ESF ligada à gravidez na adolescência. } \\
\text { Conclusão evidenciou que trabalhar com os } \\
\text { adolescentes é um grande desafio para os } \\
\text { enfermeiros do município de Divinópolis-MG, } \\
\text { pois este grupo etário quase não utiliza o serviço } \\
\text { de saúde, e que, a falta de estrutura, falta de } \\
\text { tempo (tendo em vista que há outras atividades } \\
\text { realizadas pelo enfermeiro como serviços } \\
\text { administrativos) e falta de recursos logísticos, } \\
\text { dificulta o processo de desenvolvimento de ações } \\
\text { educativas voltadas à prevenção da gravidez na } \\
\text { adolescência. }\end{array}$ \\
\hline 2017 & Assistência & Mostra de & ROCHA et al. & Evidenciar o papel do & $\begin{array}{l}\text { Resultados: O trabalho do enfermeiro neste } \\
\text { âmbito é diversificado, pois, além do cuidado ao }\end{array}$ \\
\hline
\end{tabular}




\begin{tabular}{|c|c|c|c|c|c|}
\hline & $\begin{array}{lr}\text { enfermeiro } & \text { a } \\
\text { educação sexual } & \text { dos } \\
\text { adolescentes r } & \text { na } \\
\text { atenção básica } & \end{array}$ & $\begin{array}{l}\text { Pesquisa } \\
\text { em } \\
\text { Ciência e } \\
\text { Tecnologi } \\
\text { a } 2017\end{array}$ & & $\begin{array}{lr}\text { enfermeiro } & \text { na } \\
\text { educação sexual } & \text { dos } \\
\text { adolescentes } & \text { na } \\
\text { Atenção Básica. } & \end{array}$ & $\begin{array}{l}\text { indivíduo abrange ações educativas, referentes a } \\
\text { sexualidade. Conclusão: Observa-se que o } \\
\text { enfermeiro tem papel primordial à prática de } \\
\text { ações de prevenção e promoção da saúde sexual } \\
\text { desenvolvida pela ESF. }\end{array}$ \\
\hline 2018 & $\begin{array}{lr}\text { Assistência } & \text { de } \\
\text { enfermagem } & \text { ao } \\
\text { Público adolescente } \\
\text { na atenção Primária }\end{array}$ & $\begin{array}{l}\text { Revista de } \\
\text { enfermage } \\
\text { m atual }\end{array}$ & MATOS et al. & $\begin{array}{lr}\text { Analisar as } & \text { ações de } \\
\text { enfermagem } & \text { voltadas } \\
\text { para o } & \text { público } \\
\text { adolescente } & \text { na } \\
\text { atenção básica, por } \\
\text { meio de uma revisão } \\
\text { integrativa } \\
\text { literatura. }\end{array}$ & $\begin{array}{l}\text { Resultados: Verificou-se que as ações voltadas } \\
\text { para este público, na maioria das vezes, } \\
\text { resumem-se em acolhimento e escuta do } \\
\text { paciente, isso quando este procura o serviço. } \\
\text { Conclusão: É necessária a criação de ações } \\
\text { concretas que atendam às necessidades } \\
\text { específicas desse grupo, bem como preparo da } \\
\text { equipe para lidar com esta população, } \\
\text { propiciando vinculo e atenção integral à saúde do } \\
\text { adolescente. }\end{array}$ \\
\hline 2018 & $\begin{array}{l}\text { Conhecimento sobre } \\
\text { contracepção e } \\
\text { fatores associados ao } \\
\text { planejamento de } \\
\text { gravidez na } \\
\text { adolescência }\end{array}$ & $\begin{array}{l}\text { Revista } \\
\text { cogitar } \\
\text { enfermage } \\
\mathrm{m}\end{array}$ & LAGES et al. & 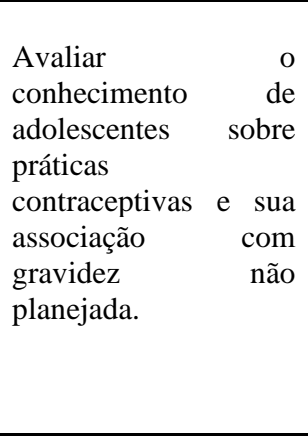 & $\begin{array}{l}\text { Resultado: a pesquisa mostrou que o baixo } \\
\text { conhecimento das práticas contraceptivas está } \\
\text { associado ao planejamento de gravidez, } \\
\text { aumentando em } 4,5 \% \text { as chances de uma } \\
\text { gravidez não planejada. } \\
\text { Conclusão: o conhecimento não é o único fator } \\
\text { responsável, mas contribui significativamente } \\
\text { para o desfecho da gravidez não planejada, } \\
\text { considerando que o fato de a adolescente não } \\
\text { saber utilizar a pílula do dia seguinte aumenta em } \\
3,93 \text { vezes a chance de ter uma gravidez não } \\
\text { planejada. }\end{array}$ \\
\hline 2018 & $\begin{array}{lr}\text { Estratégia } & \text { da } \\
\text { atenção primaria } & \text { na } \\
\text { prevenção } & \text { da } \\
\text { gestação } & \text { em } \\
\text { adolescentes: uma } \\
\text { revisão sistemática } \\
\text { de literatura } \\
\end{array}$ & $\begin{array}{l}\text { Revista } \\
\text { saúde e } \\
\text { desenvolv } \\
\text { imento }\end{array}$ & $\begin{array}{l}\text { OLIVEIRA et } \\
\text { al. }\end{array}$ & $\begin{array}{l}\text { Identificar estratégias } \\
\text { do enfermeiro na } \\
\text { atenção primaria para } \\
\text { prevenção da gravidez } \\
\text { em adolescentes }\end{array}$ & $\begin{array}{l}\text { Resultados: Dificuldade de dialogar com os } \\
\text { adolescentes sobre prevenção, e necessidade do } \\
\text { treinamento de toda a equipe para um } \\
\text { atendimento de forma clara e objetiva. } \\
\text { Conclusão: Define-se que as atribuições de } \\
\text { enfermagem, estão interligadas diretamente com } \\
\text { o diálogo dentro da casa dos adolescentes. }\end{array}$ \\
\hline 2019 & $\begin{array}{lr}\text { Gravidez } & \text { na } \\
\text { adolescência: } & \text { um } \\
\text { desafio intersetorial }\end{array}$ & $\begin{array}{l}\text { Revista } \\
\text { enfermage } \\
\text { m Brasil }\end{array}$ & $\begin{array}{l}\text { LIMA,A.V..et } \\
\text { al. }\end{array}$ & Analisar & $\begin{array}{l}\text { Resultados: Dificuldade de dialogar com os } \\
\text { adolescentes sobre prevenção, e necessidade do } \\
\text { treinamento de toda a equipe para um } \\
\text { atendimento de forma clara e objetiva. } \\
\text { Conclusão: Define-se que as atribuições de } \\
\text { enfermagem, estão interligadas diretamente com } \\
\text { o diálogo dentro da casa dos adolescentes. }\end{array}$ \\
\hline 2019 & $\begin{array}{lr}\text { Gravidez } & \text { na } \\
\text { adolescência: } & \text { uma } \\
\text { abordagem } & \\
\text { preventiva } & \text { na } \\
\text { atenção básica } & \end{array}$ & Una-sus & $\begin{array}{l}\text { MENON,R.L. } \\
\text { S. }\end{array}$ & $\begin{array}{l}\text { Diminuir a incidência } \\
\text { de gestantes menores } \\
\text { de } 18 \text { anos do } \\
\text { território da Equipe de } \\
\text { Saúde da Família } \\
\text { (ESF) da Unidade } \\
\text { Básica de Saúde } \\
\text { (UBS) Rubens } \\
\text { Antônio Baqueta } \\
\end{array}$ & $\begin{array}{l}\text { Resultados: Espera-se diminuir a incidência de } \\
\text { gestantes menores de } 18 \text { anos a médio e longo } \\
\text { prazo através da intervenção continuada, } \\
\text { seguindo os mesmos moldes das diretrizes } \\
\text { propostas em } 2011 \text { pela Organização Mundial de } \\
\text { Saúde (OMS), em parceria com o Fundo de } \\
\text { População das Nações Unidas (UNFPA), } \\
\text { portanto impossibilitado de apresentar resultados } \\
\text { concretos sobre a ação preventiva em questão. }\end{array}$ \\
\hline 2019 & $\begin{array}{lr}\text { Estratégias } & \\
\text { utilizadas } & \text { pelas } \\
\text { enfermeiras na } & \text { na } \\
\text { atenção básica } & \text { para } \\
\text { a prevenção } & \text { da } \\
\text { gravidez } & \text { na } \\
\text { adolescência } & \end{array}$ & $\begin{array}{l}\text { Revista } \\
\text { textura }\end{array}$ & $\begin{array}{l}\text { NOGUEIRA } \\
\text { et al. }\end{array}$ & \begin{tabular}{lr}
\multicolumn{2}{l}{ Analisar as estratégias } \\
desenvolvidas por \\
enfermeiras r da \\
atenção básica quanto \\
à prevenção & da \\
gravidez & na \\
adolescência em & um \\
município & do \\
recôncavo da Bahia.
\end{tabular} & $\begin{array}{l}\text { Resultados: O resultado do estudo mostrou que } \\
\text { as principais estratégias para prevenção da } \\
\text { gravidez na adolescência é a dispensação de } \\
\text { métodos contraceptivos, o que põe em evidência } \\
\text { a falta de ações como sala de espera, palestras e } \\
\text { atividades de sensibilização individuais no } \\
\text { cotidiano da atenção básica. } \\
\text { Conclusão: percebeu-se que as ações de } \\
\text { prevenção da gravidez realizadas pelas } \\
\text { enfermeiras da atenção básica encontram } \\
\text { entraves para sua efetiva concretização, sendo } \\
\text { centradas ainda na entrega de métodos } \\
\text { contraceptivos e se afastando do princípio da } \\
\text { integralidade da atenção. }\end{array}$ \\
\hline
\end{tabular}




\begin{tabular}{|c|c|c|c|c|c|}
\hline 2020 & $\begin{array}{ll}\text { Abordagem } & \text { do } \\
\text { enfermeiro } & \text { na } \\
\text { gravidez } & \text { na } \\
\text { adolescência } & \end{array}$ & $\begin{array}{l}\text { Brazilian } \\
\text { Journal of } \\
\text { health } \\
\text { Review }\end{array}$ & $\begin{array}{l}\text { FERRAZ,A.C } \\
\text {.S et al. }\end{array}$ & $\begin{array}{lr}\text { Identificar } & \text { a } \\
\text { assistência } & \text { do } \\
\text { enfermeiro diante } & \text { da } \\
\text { gravidez } & \text { na } \\
\text { adolescência e } & \text { os } \\
\text { fatores } & \text { que } \\
\text { influenciam, além de } & \text { de } \\
\text { propor um fluxograma } \\
\begin{array}{lr}\text { de atendimento do } \\
\text { enfermeiro ratéria de }\end{array} \\
\begin{array}{lr}\text { Estratégíde } \\
\text { da Família }\end{array} \\
\end{array}$ & $\begin{array}{l}\text { Resultados: Foi identificado que o tripé formado } \\
\text { pela família, a comunidade e a escola, que } \\
\text { deveriam orientar as adolescentes em suas } \\
\text { escolhas sexuais e reprodutivas, têm limitações } \\
\text { severas para exercer sua função. } \\
\text { Conclusão: É fundamental a capacitação do } \\
\text { Enfermeiro para o acolhimento e } \\
\text { acompanhamento da gestação de uma } \\
\text { adolescente. Para tal, foi proposto um fluxograma } \\
\text { para apoiar o enfermeiro no caso de gravidez na } \\
\text { adolescência na Estratégia de Saúde da Família. }\end{array}$ \\
\hline 2020 & $\begin{array}{lr}\text { Educação } & \text { sexual } \\
\text { reprodutiva } & \text { do } \\
\text { adolescente } & \text { escolar . }\end{array}$ & $\begin{array}{l}\text { Revista de } \\
\text { enfermage } \\
\text { m UFPE }\end{array}$ & $\begin{array}{l}\text { FRANCO, } \\
\text { M.S. et al. }\end{array}$ & $\begin{array}{l}\text { Relatar a experiência } \\
\text { de estudantes do curso } \\
\text { de enfermagem na } \\
\text { implementação de } \\
\text { intervenções } \\
\text { educacionais para a } \\
\text { promoção da saúde } \\
\text { sexual e reprodutiva } \\
\text { do adolescente } \\
\text { escolar. }\end{array}$ & $\begin{array}{l}\text { Resultado: Notou-se a carência no conhecimento } \\
\text { dos adolescentes escolares acerca da temática da } \\
\text { saúde sexual e reprodutiva, entretanto, a } \\
\text { intervenção no ambiente escolar mostrou ser um } \\
\text { ambiente promissor para o processo de educação } \\
\text { em saúde realizado, sobretudo, pelo enfermeiro } \\
\text { no âmbito da Estratégia Saúde da Família com } \\
\text { outros profissionais da saúde e da educação. } \\
\text { Conclusão: enfatiza-se a necessidade de } \\
\text { atividades no âmbito escolar a fim de promover o } \\
\text { conhecimento e adoção hábitos e práticas } \\
\text { saudáveis que impactem e assegurem aos } \\
\text { estudantes riscos mínimos de injúrias à saúde } \\
\text { sexual e reprodutiva }\end{array}$ \\
\hline 2020 & $\begin{array}{lr}\text { A } & \\
\text { valiação de } & \text { objeto } \\
\text { virtual rel } & \text { de } \\
\text { aprendizagem } & \text { sobre } \\
\text { pré-natal r para } \\
\text { adolescentes } \\
\text { grávidas na } \\
\text { básica }\end{array}$ & $\begin{array}{l}\text { Acta } \\
\text { paulista de } \\
\text { enfermage } \\
\text { m scielo }\end{array}$ & $\begin{array}{l}\text { FORTES, } \\
\text { S.R.; et al. }\end{array}$ & $\begin{array}{l}\text { Descrever o processo } \\
\text { de construção e } \\
\text { avaliação de um } \\
\text { Objeto Virtual de } \\
\text { Aprendizagem sobre } \\
\text { pré-natal r para } \\
\text { adolescentes grávidas } \\
\text { no contexto da } \\
\text { atenção básica. }\end{array}$ & $\begin{array}{l}\text { Resultado: Destacou-se como sugestão apontada } \\
\text { por estes juízes a melhoria do layout, que foi } \\
\text { atendida. } \\
\text { O Objeto Virtual de Aprendizagem foi avaliado } \\
\text { de forma satisfatória por juízes em Enfermagem } \\
\text { em Saúde da Mulher e Informática, quanto a } \\
\text { qualidade e ergonomia. Desta forma, acredita-se } \\
\text { que o GESTAQ possa ser usado como recurso } \\
\text { digital complementar ao processo educativo de } \\
\text { adolescentes grávidas no contexto da atenção } \\
\text { básica para fornecer orientações sobre pré-natal. }\end{array}$ \\
\hline 2020 & $\begin{array}{l}\text { Atenção integral à } \\
\text { saúde do } \\
\text { adolescente pela } \\
\text { Atenção Primária à } \\
\text { Saúde no território } \\
\text { brasileiro: uma } \\
\text { revisão integrativa }\end{array}$ & $\begin{array}{l}\text { Interface } \\
\text { Comunica } \\
\text { ção, } \\
\text { Saúde, } \\
\text { Educação }\end{array}$ & $\begin{array}{l}\text { FREITAS, } \\
\text { R.S et al. }\end{array}$ & $\begin{array}{l}\text { Sistematizar } \\
\text { experiências de } \\
\text { cuidado ao } \\
\text { adolescente pela APS. }\end{array}$ & $\begin{array}{l}\text { Resultados: Os dados foram interpretados pela } \\
\text { análise temática de conteúdo. Observaram-se } \\
\text { estigmas no cuidado ao adolescente; lacunas na } \\
\text { formação profissional; acolhimento centrado na } \\
\text { enfermagem; fragilidades dos vínculos; escassez } \\
\text { de recursos estruturais e humanos; ações } \\
\text { educativas, porém normativas; fragmentação das } \\
\text { práticas; e barreiras de acesso à rede de saúde e } \\
\text { intersetorial } \\
\text { Conclusão: Conclui-se, portanto, que, para } \\
\text { alcançar um cuidado ampliado, é preciso } \\
\text { repensar as práticas e dar voz ao adolescente. }\end{array}$ \\
\hline 2020 & $\begin{array}{l}\text { Gravidez na } \\
\text { adolescência, } \\
\text { iniciação sexual e } \\
\text { gênero: perspectivas } \\
\text { em disputa. Caderno } \\
\text { de saúde publica }\end{array}$ & $\begin{array}{l}\text { Caderno } \\
\text { de saúde } \\
\text { publica }\end{array}$ & $\begin{array}{l}\text { SILVA,C.C. } \\
\text { et al. }\end{array}$ & 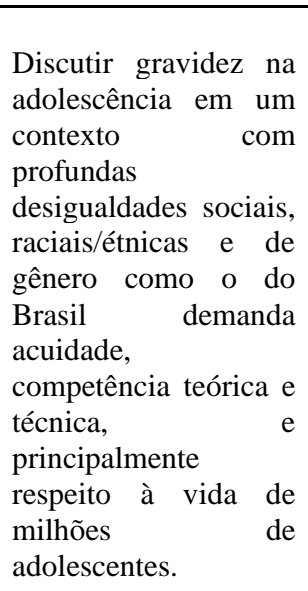 & $\begin{array}{l}\text { Resultados: } \\
\text { Revisões sistemáticas sobre o reiteram a } \\
\text { ineficácia e o desperdício de recursos públicos } \\
\text { aplicados nos programas de educação baseados } \\
\text { na premissa da abstinência sexual } \\
\text { Conclusão: Esses são elementos imprescindíveis } \\
\text { para a construção da autonomia juvenil e para o } \\
\text { exercício da sexualidade com base na perspectiva } \\
\text { do reconhecimento da alteridade e dos direitos } \\
\text { humanos. }\end{array}$ \\
\hline
\end{tabular}




\begin{tabular}{|c|c|c|c|c|c|}
\hline 2020 & $\begin{array}{l}\text { Tendência temporal } \\
\text { e fatores associados } \\
\text { à gravidez na } \\
\text { adolescência }\end{array}$ & Revista & $\begin{array}{l}\text { CASAGRAN } \\
\text { DE,M.1.1. et } \\
\text { al. }\end{array}$ & $\begin{array}{l}\text { Analisar a tendência e } \\
\text { os fatores associados } \\
\text { à gravidez na } \\
\text { adolescência em um } \\
\text { município do Paraná. }\end{array}$ & $\begin{array}{l}\text { Resultado: Associaram-se à gravidez na } \\
\text { adolescência as variáveis: estar sem } \\
\text { companheiro; escolaridade menor que oito anos; } \\
\text { primiparidade; idade gestacional menor que } 37 \\
\text { semanas; cesárea; número de consultas menor } \\
\text { que sete; e Apgar menor que sete no primeiro } \\
\text { minuto. } \\
\text { Conclusão: A análise dos dados permitiu } \\
\text { conhecer a tendência e os fatores associados à } \\
\text { gravidez na adolescência, sugerindo situações de } \\
\text { risco que podem decorrer deste evento. }\end{array}$ \\
\hline
\end{tabular}

Fonte: Autores.

Após a leitura dos artigos selecionados, emergiu-se duas categorias: Ações do enfermeiro para auxiliar na prevenção a gravidez na adolescência e Orientações do enfermeiro para sensibilização da prevenção a gravidez na adolescência.

\section{Categoria 1. Ações do enfermeiro para auxiliar na prevenção a gravidez na adolescência.}

As principais estratégias para prevenção da gravidez na adolescência. É a dispensação de métodos contraceptivos o que põe em evidência a falta de ações como a sala de espera, palestra e atividades de sensibilização individual no cotidiano da atenção básica. A captação de adolescente para consulta ocorre na sua maior parte por busca ativa com agente comunitário de saúde (Costa et al., 2019).

Define-se que as atribuições de enfermagem, estão interligadas diretamente com o diálogo dentro da casa dos adolescentes, onde pode estar ocorrendo o incentivo da prevenção cada vez antes do início das relações sexuais dos jovens, ficou evidenciado a necessidade do enfermeiro na criação de estratégias na atenção primária, como objetivos foram alcançados, e seus resultados, através desta investigação apresentam relevância social, profissional e acadêmica (Oliveira et al., $18)$.

Vale a pena ressaltar que o acesso do adolescente a unidade de saúde deve ser facilitado e o mesmo deve ser visto como indiví-duo que necessita de atenção integral, privacidade e confidencialidade pelos profissionais de saúde, além de acolhido de forma adequada (Nogueira et al., 2019).

A Estratégia de Saúde da Família e o Programa Saúde na Escola são instrumentos essenciais para aproximação desta população, criação de vínculo e queda de barreiras, trazendo o adolescente para dentro da unidade de saúde. Entretanto, apesar dos esforços, muitas vezes as ações realizadas parecem insuficientes para a prevenção da gravidez na adolescência. Talvez realmente sejam, pois, além de discutir saúde sexual e reprodutiva, um tema essencial deve ser trabalhado, o empoderamento das adolescentes, capacitando-as para a tomada de decisões, para a realização de projetos e planos, para a continuidade dos estudos (Casagrande et al., 2020).

As considerações finais desses estudos apontam para a necessidade de ações educativas sobre saúde sexual e sexualidade para possibilitar as adolescentes à oportunidade de escolhas de métodos anticoncepcionais que lhes permitam ter vida sexual sem correr o risco de uma gravidez indesejada. Considerou-se também importante a capacitação dos profissionais das equipes de saúde da família na forma de lidar com esse segmento da sociedade que ainda procura pouco os serviços de saúde (Pereira et al.,2016).

\section{Categoria 2. Orientações do enfermeiro para sensibilização da prevenção a gravidez na adolescência.}

Observa-se a necessidade de melhorar as informações sobre a prevenção da gestação indesejada, tendo em vista que a gravidez precoce contribui para o aumento de intercorrências obstétricas e/ou neonatais, além de repercussões sociais na vida 
dos adolescentes e sua família. Esse trabalho tem por objetivo elaborar uma proposta de intervenção que contribua para diminuir a incidência de gravidez indesejada junto as adolescentes dessa área de abrangência (Santiago et al., 2016).

Um fato considerável atualmente é que estas meninas possuem conhecimento em relação aos métodos contraceptivos, principalmente do preservativo masculino e anticoncepcional hormonal oral, porém somente o conhecimento não se traduz em uso, e para que ele ocorra faz-se necessário assegurar o acesso aos métodos e o combate às barreiras que prejudicam a sua utilização (Santos et al., 2019).

Frente a este painel, há três pilares essenciais para a prevenção da gravidez na adolescência: a escola, a família e a atenção básica. Para o sucesso das ações é necessário que o trabalho ocorra de forma intersetorial. A escola é um local privilegiado para a execução de ações de educação em saúde, pois é um espaço para aquisição de conhecimento, socialização e debates, porém vê-se a necessidade de discutir a saúde sexual e reprodutiva de forma cada vez mais precoce (Vicentim et al., 2018).

O tema da sexualidade adolescente gerou intenso debate na sociedade brasileira em janeiro de 2020, com a proposta de abstinência (ou adiamento da vida sexual) como política pública para o enfrentamento da gravidez na adolescência.

Discutir gravidez na adolescência em um contexto com profundas desigualdades sociais, raciais/étnicas e de gênero como o do Brasil demanda acuidade, competência teórica e técnica, e principalmente respeito à vida de milhões de adolescentes. Esses são sujeitos de direitos, em processo de aprendizado da autonomia, do cuidado e controle de si e da sexualidade, como dimensão inerente às relações sociais.

O tópico entrou em pauta após anúncio do Ministério da Mulher, da Família e dos Direitos Humanos (MMFDH) sobre a elaboração de uma Política Nacional de Prevenção ao Risco da Atividade Sexual Precoce (Silva, 2020).

Com a criação da Semana Nacional de Prevenção da Gravidez na Adolescência, pelo Governo Federal, por intermédio da Lei $\mathrm{n}^{\circ}$ 13.7981, em janeiro de 2019, a ser comemorada anualmente na primeira semana de fevereiro, o MMFDH, em parceria com o Ministério da Saúde, iniciou uma campanha nacional de sensibilização de adolescentes sobre os efeitos indesejáveis da "gravidez precoce". Embora o termo "abstinência sexual" não tenha sido adotado, possivelmente pela enorme rejeição e fortes críticas recebidas (Brasil, 2015).

\section{Considerações Finais}

Finaliza-se que o enfermeiro e primordial a frente da estratégia de saúde da família pois ele desempenha e desenvolve estratégias de prevenção a gravidez na adolescência, tendo em vista que o trabalho do enfermeiro neste âmbito é diversificado, pois, além do cuidado ao indivíduo abrange ações educativas, referentes a sexualidade.

Contudo, o presente estudo evidenciou que os adolescentes precisam iniciar a educação sexual o mais cedo possível, cabendo aos pais e sendo assumida pela escola e por profissionais de saúde, com tudo o enfermeiro tem o papel principal na orientação dos adolescentes promovendo palestra, roda de conversas, atividades educativas.

Notou-se que as adolescentes que engravidaram tendem a ser excluídas afastada de seus grupos e pode resultar em complicações como abandono das atividades, risco de morte e complicações para mãe e recém-nascido.

Sendo assim, espera-se que a pesquisa possibilite um maior conhecimento sobre a prevenção da gravidez na adolescência, estimulando uma reflexão profunda, neste cenário, visando que os profissionais de enfermagem tenham uma melhor compreensão e um olhar diferenciado sob essa ótica, agregando de maneira positiva em toda sua atuação profissional.

Bem como, contribuir com a literatura, servindo como fonte de consulta bibliográfica para futuros profissionais de enfermagem, e também, como base para outras produções científicas, corroborando a esse contexto, proporcionando um aumento das discussões para que melhores práticas sejam implementadas. Gerando grandes repercussões também, para toda 
sociedade, uma vez que, a pesquisa é um meio de compartilhamento de informações pertinentes à saúde, buscando compreender a atuação profissional, e com isso, estimular reflexões críticas acerca da temática.

\section{Referências}

Araújo, A. K. L., \& Nery, I. S. (2018). Conhecimento sobre contracepção e fatores associados ao planejamento de gravidez na adolescência. Cogitare Enfermagem, 23(2), 321-312.

Arbelo, M. L. (2016). Intervenção educativa sobre gravidez na adolescência. BRASIL. Lei 8.069, de 13 de julho de 1990 . Estatuto da Criança $e$ do Adolescente. Brasília: Ministério da Justiça, 15, 123-129.

Borrell, M. M. Intervenção educativa: prevenção da gravidez na adolescência na Estratégia de Saúde da Família do PSF Pedra Solta, Itiúba-BA. Universidade Federal do Maranhão (UFMA), 15 (3), 11-16.

Cabral, C. D. S. \& Brandão, E. R. (2020). Gravidez na adolescência, iniciação sexual e gênero: perspectivas em disputa. Cadernos de Saúde Pública, 36.

Lopes, M. C. D. L., Oliveira, R. R. D., Silva, M. D. A. P. D., Padovani, C., Oliveira, N. L. B. D. \& Higarashi, I. H. (2020). Tendência temporal e fatores associados à gravidez na adolescência. Revista da Escola de Enfermagem da USP, 54.

Costa, R. S. N., Fonseca, I. P., Barreto, F. L., Oliveira, M. T. C. S. \& Lopes, M. V. G. (2019). Estratégias utilizadas pelas enfermeiras na atenção básica para a prevenção da gravidez na adolescência. Textura, 13(22), 218-227.

Fiedler, M. W., Araújo, A. \& Souza, M. C. C. D. (2015). A prevenção da gravidez na adolescência na visão de adolescentes. Texto \& ContextoEnfermagem, 24, 30-37.

Fonseca, J. M. (2019). Assistência de enfermagem às adolescentes grávidas. Revista Científica Multidisciplinar Núcleo do Conhecimento, 9(3), 135-184

Franco, M. D. S., Barreto, M. T. S., Carvalho, J. W. D., Silva, P. P. D., Moreira, W. C., Cavalcante, M. C. \& Lima, L. H. D. O. (2020). Educação em saúde sexual e reprodutiva do adolescente escolar. Rev. enferm. UFPE on line, 8(1), 123-145.

Silva, R. F. \& Engstrom, E. M. (2020). Atenção integral à saúde do adolescente pela Atenção Primária à Saúde no território brasileiro: uma revisão integrativa. Interface-Comunicação, Saúde, Educação, 24, 212-243.

Garcia, A. R. \& Lúcia, A. K. 2016). Gravidez na adolescência e o papel do enfermeiro. Bvsalud. 23(4). 234-244.

Gurgel, M. G. I., Alves, M. D. S., Moura, E. R. F., Pinheiro, P. N. D. C. \& Rego, R. M. V. (2010). Desenvolvimento de habilidades: estratégia de promoção da saúde e prevenção da gravidez na adolescência. Revista Gaúcha de Enfermagem, 31, 640-646.

Leal, M. C. B., Porto, A. O., de Brito Barbosa, C., Fernandes, T. S. S., Pereira, E. S. \& Viana, T. B. P. (2018). Assistência de enfermagem ao público adolescente na atenção primária. Revista Enfermagem Atual In Derme, 86(24).

Lopes, M. C. D. L., Oliveira, R. R. D., Silva, M. D. A. P. D., Padovani, C., Oliveira, N. L. B. D. \& Higarashi, I. H. (2020). Tendência temporal e fatores associados à gravidez na adolescência. Revista da Escola de Enfermagem da USP, 54.

MENON, R. L. D. S. (2019). Gravidez na adolescência: uma abordagem preventiva na Atenção Básica de Saúde. 23(3), $345-365$.

Almeida, T. M., \& Rocha, L. S. (2015). Gravidez na adolescência: reconhecimento do problema para atuação do enfermeiro na sua prevenção. Anais Simpac, 7(1).

Reis, K. O., da Silva Ferreira, W. F. \& Da Silva, A. (2018). Estratégias da atenção primaria na prevenção da gestação em adolescentes: uma revisão sistemática de literatura. Revista Saúde e Desenvolvimento, 12(11), 117-152.

Oliveira, T. C. D., Carvalho, L. P., \& Silva, M. A. D. (2008). O enfermeiro na atenção à saúde sexual e reprodutiva dos adolescentes. Revista Brasileira de Enfermagem, 61(3), 306-311.

Pereira, K. R. (2016).Gravidez na adolescência um desafio para o enfermeiro: revisão de literatura. Unasus. 34(15). 354-9-376

Rocha, M. C. D. J. (2013). Gravidez na adolescência: a importância do enfermeiro como educador-proposta de intervenção no município de Buritis-Minas Gerais. 24, 567-581.

Rocha, M. K., Silva, C. T., Maciel, L. M. N. L., Bezerra, A. C. S., Clemente, M. H. S. \& Vasconcelos, C. (2013). Assistência do enfermeiro a educação sexual dos adolescentes na atenção básica. Ibmec, 8(2), 344-456.

Santiago, R. F., Andrade, E. M. L. R., Mendes, I. A. C., Viana, M. C. A., \& Nery, I. S. (2020). Avaliação de objeto virtual de aprendizagem sobre pré-natal para adolescentes grávidas na atenção básica. Acta Paulista de Enfermagem, 33.

Santos, A. C. F., Vador, R. M. F., Cunha, F. V., \& De Almeida, A. (2020). Abordagem do enfermeiro na gravidez na adolescência. Brazilian Journal of Health Review, 3(6), 17438-17456.

Silva, L. S. A. H., \& de Melo Tavares, C. M. (2017). Recovery: É possível cuidar nesta perspectiva em um hospital psiquiátrico. Revista Pró-UniverSUS, 8(2), 76-78. 
Research, Society and Development, v. 11, n. 2, e6911225479, 2022

(CC BY 4.0) | ISSN 2525-3409 | DOI: http://dx.doi.org/10.33448/rsd-v11i2.25479

Taborda, J. A., Silva, F. C. D., Ulbricht, L., \& Neves, E. B. (2014). Consequências da gravidez na adolescência para as meninas considerando-se as diferenças socioeconômicas entre elas. Cadernos Saúde Coletiva, 22, 16-24.

Vicentim, A. L., dos Santos, N. S. G. M., \& Santos, M. D. L. S. G. (2019). Gravidez na adolescência: um desafio intersetorial. Enfermagem Brasil, 18(5), 610611.

Vieira, K. P. \& Medrato, B. A. P. (2016). A prevenção da gravidez não desejada em adolescentes: projeto de intervenção na atenção primária, 24(9), 220-213.

Wildemberg, F. M. W., Araújo, A. \& Souza, M. C. C. D. (2015). A prevenção da gravidez na adolescência na visão de adolescentes. Texto \& ContextoEnfermagem, 24, 30-37. 